\title{
Generation and Selection of a Panel of Pan-Filovirus Single-Chain Antibodies using Cell-Free Ribosome Display
}

\author{
Adinarayana Kunamneni, ${ }^{1,2}$ Elizabeth C. Clarke, ${ }^{1}$ Chunyan Ye, ${ }^{1}$ Steven B. Bradfute, ${ }^{1}$ and Ravi Durvasula ${ }^{1,2 \star}$ \\ ${ }^{1}$ Department of Internal Medicine, Center for Global Health, University of New Mexico, Albuquerque, New Mexico; ${ }^{2}$ Department of Medicine, \\ Loyola University Medical Center, Chicago, Illinois
}

\begin{abstract}
Filoviruses, which include ebolaviruses and marburgvirus, can cause outbreaks of highly lethal hemorrhagic fever. This disease causes significant morbidity and mortality in humans and non-human primates, with human fatality rates reaching $90 \%$ during some outbreaks. Currently, there is lack of licensed vaccines or antivirals for these viruses. Since early symptoms of filovirus infection mimic more common diseases, there is a strong unmet public health and biodefense need for broad-spectrum filovirus rapid diagnostics. We have generated a panel of mouse single-chain Fvantibodies (scFvs) to filovirus glycoproteins (GPs) using cell-free ribosome display and determined their cross-reactivity profiles to all known filovirus species. Two scFvs (4-2 and 22-1) were able to detect all known Ebolavirus and Marburgvirus species. This is the first report on ribosome display scFvs that can detect a broad set of filovirus GPs, which demonstrates the potential for use in diagnostics.
\end{abstract}

\section{INTRODUCTION}

The recent Ebola virus pandemic was a health crisis with global repercussions (PMID: 27813879). Rapid spread of the disease within the epidemic regions coupled with migration of infected persons has underscored the need for rapid, robust, and inexpensive diagnostic tools. There are six filoviruses known to be pathogenic in humans: four ebolaviruses (Ebola [EBOV], Sudan [SUDV], Tai Forest [TAFV], and Bundibugyo [BDBV]) and two marburgviruses (Ravn [RAW] and Marburg [MARV]) (PMID: 21046175). Presently, diagnostics for active infection are largely based on realtime PCR assays. Although these are rapid, highly sensitive and specific detection, these methods are expensive and require an instrument with hands-on preparation for proper use. There are ELISA-based detection platforms for EBOV, SUDV, and BDBV VP40, but sensitivity and specificity are low relative to PCR-based assays. ${ }^{1}$ Furthermore, most of the diagnostic platforms of all types have centered on detection of EBOV. There are fewer readily available monoclonal antibodies for SUDV, BDBV, TAFV, MARV, and RAW, ${ }^{2}$ severely limiting the development of antibody-based diagnostics. The WHO has recently established ideal guidelines for Ebola virus diagnostics, which include ease of handling, portability, and rapid results. ${ }^{3}$ In 2014, the FDA issued nine emergency use authorizations for EBOV diagnostics. Of these, eight were PCR based and one was an antigen ELISA with variably low sensitivity and low specificity. ${ }^{1}$ Additional concems exist regarding filovirus mutants, which may not be detected by standard assays. The recent EBOV Makona strain had mutated so that multiple known antibody epitopes had changed. ${ }^{4,5}$ This would invalidate certain diagnostic platforms that cannot rapidly be modified to detect emerging viral mutants. In addition, in the presence of selective pressure, filoviruses have been shown to readily mutate. ${ }^{6-10}$ There is, thus, a strong unmet public health and biodefense need for broad-spectrum rapid diagnostics and outbreak control.

Ribosome display is a powerful tool for selecting specific antibodies from a large library in a cell-free system. ${ }^{11-13}$ It involves the generation of stable antibody-ribosome-mRNA (ARM) ternary complexes, followed by panning of the ARM

\footnotetext{
*Address correspondence to Ravi Durvasula, Department of Medicine, Loyola University Medical Center, 2160 S 1st Ave., Chicago, IL 60153-5590. E-mail: ravi.durvasula@lumc.edu
}

ternary complexes against an immobilized ligand. The mRNA encoding selected target-binding library members is then recovered as DNA from the bound ternary complexes using in situ reverse transcription polymerase chain reaction (RTPCR) and either analyzed directly by sequencing for Escherichia coli expression to screen for desirable candidates or subjected to subsequent rounds of panning for enriching ligand-specific binding molecules. The use of in situ RT-PCR also facilitates automation of the entire ribosome display process. Ribosome display has been explored to rapidly produce novel molecules from very large libraries, in combination with next-generation sequencing technology. This technology can also be combined with DNA mutagenesis for antibody evolution in vitro to produce high-affinity antibodies. More recently, ribosome display has been used to obtain ligands with sub-picomolar affinities for the relevant antigen, outperforming the affinities of most conventional mAbs. ${ }^{14}$ In addition to antibodies, ribosome display has been explored to select for scaffolds, peptides, ligand-binding molecules, receptors, enzymes, membrane proteins, and engineered vaccines. ${ }^{15}$ It can also be combined with protein microarrays to allow library-versus-library screening for high-throughput generation of antibodies and genomic discovery of protein-protein interactions. ${ }^{16,17}$

Single-chain Fvs (scFvs) are recombinant antibody fragments, which consist of a full variable (epitope-binding) region of an immunoglobulin heavy chain $(\mathrm{VH})$ and the corresponding variable region of an immunoglobulin light chain (VL) variable domains that are connected by a flexible peptide linker. ${ }^{18-20}$ They represent the smallest functional antigen-binding domain of an antibody necessary for high-affinity binding of an antigen. ${ }^{21}$

In this study, we report the use of a rapid, cell-free ribosomal display system to isolate a panel of scFvs that can bind the glycoprotein (GP) of EBOV, SUDV, Reston virus (RESTV), BDBV, TAFV, and MARV. The resulting antibodies have been characterized in a range of in vitro assays, which demonstrates their diagnostic potential.

\section{MATERIALS AND METHODS}

Ethics statement. Mice experimental procedures were approved by the Institutional Animal Care and Use Committee 
(IACUC) at the University of New Mexico. Animal research performed at the University of New Mexico was conducted under an IACUC-approved animal protocol (protocol \#15200336-HSC) in compliance with the Animal Welfare Act and other federal statutes and regulations relating to animals and experiments involving animals and adheres to principles stated in the Guide for the Care and Use of Laboratory Animals. ${ }^{22}$ The University of New Mexico is fully accredited by the Association for the Assessment and Accreditation of Laboratory Animal Care International.

Plasmids, strains, and reagents. All reagents used in the study were commercially available and were of reagent grade or better. All restriction enzymes and DNA modification enzymes were of molecular biology grade. All primers were purchased from Invitrogen (Carlsbad, CA). pGEM ${ }^{\circledR}-\mathrm{T}$ easy cloning vector (Cat. \#A1360) and TNT T7 Quick for PCR DNA kit (rabbit reticulocyte cell-free extract, Cat. \#L5540) were purchased from Promega (Madison, WI). Plasmid pBAK1, previously constructed in our laboratory ${ }^{23}$ is based on the pET-26b vector (Novagen, Madison, WI). Rabbit anti-Strep tag II polyclonal antibody, affinity purified horseradish peroxidase (HRP) conjugated (Cat. \#A01742-100); mouse anti-his antibody (Cat. \#A00186-100); and rabbit anti-mouse AP antibody (Cat. \#SAB3701085) were purchased from GenScript Inc. (Piscataway, NJ), Invitrogen, and Sigma-Aldrich (St. Louis, MO), respectively. The AP detection reagent kit (Cat. \#692643) was purchased from Novagen.

Cell lines and plasmids. HEK293T cells (American Type Culture Collection) were maintained in Dulbecco's Modified Eagle Medium (Gibco, Cincinnati, $\mathrm{OH}$ ) $+10 \%$ fetal bovine serum $+1 \%$ penicillin/streptomycin. Filovirus GPs were encoded in pcDNA3.1 plasmids.

Ebola viruslike particles (VLPs). Ebola viruslike particles were prepared essentially as previously described, with minor modifications. $^{24,25}$ Plasmids expressing EBOV-Yambuku GP1,2 and VP40 were transfected into 293T cells using jetPRIME, according to the manufacturer's protocol. Three days after transfection, supernatants were harvested and centrifuged at $9,000 \times g$ for 2 hours. VLP pellets were resuspended in phosphate buffered saline (PBS) and layered for a discontinuous 60/30/10 gradient, and ultracentrifuged for 18 hours at $150,000 \mathrm{~g}$. Lower bands were collected, diluted in PBS, and centrifuged for 2 hours at $100,000 \times g$. Pellets were resuspended in PBS and quantitated.

Immunization of mice. C57BL/6 mice were obtained from The Jackson Laboratory (Ellsworth, ME). Mice were vaccinated i.p. with 10 ug of EBOV VLPs mixed with alum twice at 3week intervals. Splenocytes of individual mice were placed in $10 \mathrm{~mL}$ of TRIzol for use in RNA isolation approximately 6 weeks after the last vaccination.

Generation of single-chain antibodies by cell-free ribosome display. Antibody library construction. Total spleen RNA was prepared as described by Kunamneni et al. ${ }^{13}$ Mice spleens were minced and homogenized in $10 \mathrm{~mL}$ of TRlzol (Invitrogen). The total RNA pellet was air-dried and resuspended in $500 \mu \mathrm{L}$ of nuclease-free water (stored at $-80^{\circ} \mathrm{C}$ ). Complementary DNA (cDNA) was synthesized from approximately $25 \mu \mathrm{g}$ of total RNA using a SuperScript II RT (Invitrogen) following the manufacturer's instructions provided.

For antibody library construction, the PCR primers were based on published sequences ${ }^{26}$ with minor modifications (see Supplemental Table 1). The primers were designed to introduce in-frame $\mathrm{Ncol}$ and Notl restriction sites to the $5^{\prime}$ end of the $\mathrm{VH}$ sequence and to the $3^{\prime}$ end of the $\mathrm{VL}$ sequence, respectively. The VH_FNH_R and VL_F/VL_R sets of primers (see Supplemental Table 1) were used for PCR amplification of $\mathrm{VH}$ and $\mathrm{VL}$ gene segments using the cDNA template, respectively. The VH_R and VL_F set of primers (see Supplemental Table 1) were used to introduce overlapping sequences which enabled the scFv gene fragments to be assembled by overlap extension PCR, and these primers encode a 20 -amino acid linker sequence $\left(\mathrm{G}_{4} \mathrm{~S}\right)_{4}$. The amplified heavy- and light-chain products were purified and pooled, and an aliquot of light-and heavy-chain templates was subjected to overlap extension PCR amplification using Link to introduce the Strep II tag and Kozak sequence on the $5^{\prime}$ end and an overlap extension on the $3^{\prime}$ end to facilitate joining to the variable heavy-chain libraries using MKR and KzSTREPII. Finally, the PCR product encoding all the variable heavy-chain and light-chain combinations was amplified with primers RDT7 and MKR to introduce the T7 site into the Strep tag II-conjugated VH-VL library and to produce the DNA encoding the anti-Ebola immunoglobulin scFv libraries. The initial PCR amplification reactions were performed at a $52^{\circ} \mathrm{C}$ annealing temperature with 30 cycles, and the subsequent library assembly step used 16 cycles with GoTaq DNA polymerase and 20 pmoL of each primer pair per reaction. DNA fragments were resolved by gel electrophoresis on $1 \%$ (wt/vol) agarose gels. DNA isolation from agarose gels was carried out following QIAquick DNA gel purification kit instructions. The final purified PCR product $\sim 0.9 \mathrm{~kb}$ is a template for ribosome display.

Cell-free ribosome display technology. To select specific antibody fragments, we have used a modified eukaryotic ribosome display with slight modifications. ${ }^{27}$ The in vitro transcription and translation reaction was based on a coupled rabbit reticulocyte lysate system (Promega's TNT quickcoupled transcription-translation system) and performed according to the supplier's protocol. The PCR-generated DNA library of antibody-coding genes derived from mouse 1 was expressed in this lysate system. Briefly, $50 \mu \mathrm{L}$ of the transcription/translation mixture containing $40 \mu \mathrm{L}$ of TNT T7 Quick Master Mix, $2 \mu \mathrm{L}$ of DNA library $(0.1-1.0 \mu \mathrm{g}), 1 \mu \mathrm{L}(1 \mathrm{mM})$ of methionine, $1 \mu \mathrm{L}$ of DNA enhancer, and $6 \mu \mathrm{L}$ of water was added, and the reaction mixture was incubated at $30^{\circ} \mathrm{C}$ for 90 minutes. Then, $5 \mu \mathrm{L}$ of RNase-free DNase I (Roche) $(2,000 \mathrm{U} / \mathrm{mL})$ was added, and the mixture was incubated for 20 minutes at $30^{\circ} \mathrm{C}$ (to remove the DNA template so that subsequent PCR only picks up pulled down RNA sequences).

To select specific antibody fragments, the $0.5-\mathrm{mL}$ PCR tubes were coated with $1 \mu \mathrm{g} / \mathrm{mL}$ of the recombinant Zaire Ebov GP in $100 \mu \mathrm{L}$ PBS at $4^{\circ} \mathrm{C}$ overnight. Protein-coated tubes were washed with PBS and blocked with $100 \mu \mathrm{L}$ of molecular biology-grade bovine serum albumin (BSA) in PBS (10 mg/mL) (New England Biolabs) for 1 hour at room temperature. The translation/transcription mixture (containing the protein-ribosome-mRNA complexes) was added to the washed and blocked protein-coated tubes and incubated on ice for 1 hour. The PCR tubes were washed three times with ribosome display washing buffer (PBS containing $0.01 \%$ Tween 20, 5 mM magnesium acetate, and 0.1\% BSA; $\mathrm{pH} 7.4$ ) and quickly washed two times with ice-cold RNase-free water, and the retained RNA (antibody sequences) was subjected to the following recovery process: in situ single-primer RT-PCR 
recovery was performed in the PCR tubes carrying selected ARM complexes using a SuperScript II reverse transcriptase (Invitrogen). The obtained cDNA was amplified in a $25-\mu \mathrm{L}$ PCR mixture for 35 cycles of 30 seconds at $94^{\circ} \mathrm{C}, 30$ seconds at $52^{\circ} \mathrm{C}, 1$ minute at $72^{\circ} \mathrm{C}$, and 10 minutes at $72^{\circ} \mathrm{C}$ with MKR and KzStrep II using GoTaq DNA polymerase (Promega). The RTPCR product from a single round of ribosome display was purified by agarose gel electrophoresis.

Cloning, expression, and purification of an anti-Ebov GP scFvs. The RT-PCR product from a third round of ribosome display was cloned into the pGEM-T Easy vector (T-Cloning ${ }^{\circledR}$ Kit, Promega) according to the manufacturer's instructions. The ligation products were transformed in pGEM-T Easy vector $E$. coli cells, and positive colonies were chosen by blue-white selection and confirmed by DNA sequencing using T7 and SP6 standard primers (Supplemental Table 1). Based on the sequencing results, the clones in the right reading frame without stop codon were chosen for prokaryotic expression. EbovGP-scFv was expressed in the form of $\mathrm{C}$-terminal $6 \mathrm{xH}$ is fusion from the prokaryotic expression vector pBAK1, previously constructed in our laboratory based on the pET-26b vector (Novagen). This vector has a strong T7 promoter and is designed to work with Rosetta-gami (DE3) strains of E.coli. The protein was induced and expressed at room temperature $\left(25^{\circ} \mathrm{C}\right)$ to increase the portion of the correctly folded, soluble EbovGP-scFv.

For the construct of scFvs, DNA was amplified with the forward primer, KzStrepll 5'CGAATTCCACCATGGCCTG GAGCCATCCGCAGTTCGAGAAGACCGGCAGCGG3' (with an $\mathrm{Ncol}$ restriction sequence highlighted in bold) and the reverse primer, MKR_Notl 5'AGTGCGGCCGCAGAACACTC ATCCTGTTGAAGCTCTTGACAATGGGTGAAGTTG3' (with an Notl restriction sequence highlighted in bold). Both set of primers allow these two amplicons (Supplemental Figure 2) to be subcloned into a pBAK1-His vector ${ }^{23}$ and were transformed into a Rosetta-gami B (DE3) E. coli strain and plated onto an LB agar plate with $100 \mu \mathrm{g} / \mathrm{mL}$ of carbenicillin and grown at $37^{\circ} \mathrm{C}$ for 16 hours. The molecular weight and isoelectric points were predicted using ExPASy bioinformatics resource portal (http://web.expasy.org/compute_pi/). ${ }^{28}$ Next day, five colonies were inoculated in $3 \mathrm{~mL}$ of $\mathrm{LB}$ media with the same antibiotics and grown at $37^{\circ} \mathrm{C}$ with $225 \mathrm{rpm}$ shaking for 16-18 hours, and the positive clones were selected by restriction analysis with $\mathrm{Ncol}$ and Notl and confirmed by DNA sequencing. The 3-mL overnight culture was used to prepare a glycerol bacterial stock and inoculated into $200 \mathrm{~mL}$ of LB medium containing the same antibiotics and grown at $37^{\circ} \mathrm{C}$ with shaking at $225 \mathrm{rpm}$ until $\mathrm{OD}_{600}$ reached between 0.4 and 0.6 . The culture was briefly chilled on ice to $25^{\circ} \mathrm{C}$, and the cells were induced by the addition of isopropyl $\beta$-D-1-thiogalactopyranoside (IPTG) (final concentration $1 \mathrm{mM}$ ) and were incubated for 12 hours at $25^{\circ} \mathrm{C}$ with shaking. Cells were harvested in $4 \times 50-\mathrm{mL}$ tubes by centrifugation at $4,000 \times \mathrm{g}, 4^{\circ} \mathrm{C}$ for 20 minutes. Cell pellets were frozen and stored at $-80^{\circ} \mathrm{C}$ before undergoing further processing.

The 50-mL cell pellet from the 200-mL culture was resuspended in $3 \mathrm{~mL}$ of lysis buffer $(20 \mathrm{mM}$ Tris- $\mathrm{HCl}, 500 \mathrm{mM} \mathrm{NaCl}$, $20 \mathrm{mM}$ Imidazole, $0.1 \%$ Triton $\mathrm{X}-100 \mathrm{pH}$ 8.0). Cells were lysed by sonication on ice $(6 \times 30$ seconds) and were centrifuged at $14,000 \times g$ for 15 minutes to remove cellular debris. The soluble fraction was filtered through 0.2- $\mu \mathrm{m}$ filters and applied to a HisTrap excel 1-mL column (GE Life Sciences Pittsburgh,
PA). The column was equilibrated and washed with $20 \mathrm{mM}$ Tris- $\mathrm{HCl}, 500 \mathrm{mM} \mathrm{NaCl}$, and $20 \mathrm{mM}$ Imidazole $\mathrm{pH}$ 8.0, and the sample was eluted with $20 \mathrm{mM}$ Tris- $\mathrm{HCl}, 500 \mathrm{mM} \mathrm{NaCl}$, and $500 \mathrm{mM}$ Imidazole, $\mathrm{pH} 8.0$ in one elution step. The purification was carried out at a constant flow of $1 \mathrm{~mL} / \mathrm{min}$. Fractions of $1 \mathrm{~mL}$ were collected through the elution step. The purified protein fractions were kept at $4^{\circ} \mathrm{C}$.

Western blot of scFv fragments. The proteins were separated by SDS-PAGE and were blotted onto polyvinylidene difluoride (PVDF) membranes using an iBlot2 Gel Transfer Device (Life Technologies, Carlsbad, CA) for 7 minutes. The membranes were blocked in $5 \% \mathrm{w} / \mathrm{v}$ skimmed milk powder/ TBS buffer at room temperature for 1 hour and then incubated with mouse anti-His antibody (GenScript) at a dilution of 1: 5,000 for 1 hour. After incubation, the membrane was washed three times with Tris buffered saline with Tween-20 (TBST) buffer, HRP-conjugated Rabbit anti-strep tag II antibody (diluted 3/10,000 in TBST) (GenScript) at room temperature for 1 hour, followed by washing with TBS-T buffer $3 \times$ for 10 minutes each. The protein was detected and visualized with ECL detection reagent (Amersham, Piscataway, $\mathrm{NJ}$ ) following the manufacturer's recommendation.

Transfections. All transfections were performed in HEK293T cells seeded in a 6-well plate (PMID: 29118454). Cells were transfected with filovirusGPs (sequences listed in PMID: 29118454) using jetPRIME according to the manufacturer's recommendations. Cells were stained with antibodies 48 hours after transfection.

Analysis of purified scFvs. Specificity, cross-reactivity, and affinity of GP-specific scFvs. To determine binding and cross-reactivity and half-maximal effective concentrations, or EC50s, scFvs were tested for binding to the GP antigens $(1 \mu \mathrm{g} /$ $\mathrm{mL}$ ) of EBOV, SUDV, BDBV, and RESTV, and to MARV Ravn GP a concentration range of $30-0.1 \mu \mathrm{g} / \mathrm{mL}$. ELISAs were performed as follows: Nunc Polysorp ELISA plates were coated overnight at $4^{\circ} \mathrm{C}$ either with target filovirus GP antigens, blocked with $2 \%$ BSA at $37^{\circ} \mathrm{C}$ for 1 hour, and incubated with anti-EBOV scFvs in $1 \%$ BSA. Commercial monoclonal (mouse anti-EBOV antibody 6D8 and mouse anti-SUDV Ebola virus antibody) and polyclonal (rabbit anti-RESTV GP, rabbit anti-BDBV GP, rabbit anti-MARV GP, and rabbit anti-TAFV GP) antibodies served as positive controls, whereas mouse anti-PilB scFv21 antibody (data not shown) served as the negative control. After 1 hour of incubation at $37^{\circ} \mathrm{C}$, plates were washed as described above and scFv was detected with 6:10,000 dilution of the HRP-conjugated Rabbit anti-strep tag II polyclonal antibody in PBS containing 1\% BSA and incubated at $37^{\circ} \mathrm{C}$ for 1 hour. After washing and tap drying, $100 \mu \mathrm{L}$ of the TMB substrate was added to each well and the plate was allowed to incubate for $15-30$ minutes at $25^{\circ} \mathrm{C}$. The reaction was stopped with $0.18 \mathrm{M} \mathrm{H}_{2} \mathrm{SO}_{4}$, and the absorbance reading was measured at $450 \mathrm{~nm}$; these values were transformed using Softmax Pro (7.1) 4 parameter curve-fit (Spectramax M3, Molecular Devices Corp., San Jose, CA).

Western blot analysis. Different purified pan-Ebola and panfilovirus antigens (Lloviu virus [LLOV], Zaire ebolavirus (ZEBOV), SUDV, RESV, BDBV, MARV, and TAFV GPs) of $1 \mu \mathrm{g} /$ $\mathrm{mL}$ were separated on $4-12 \%$ sodium dodecyl sulfate polyacrylamide gel electrophoresis (SDS-PAGE) and transferred onto PVDF transfer membranes using an iBlot2 Gel Transfer Device (Life Technologies) for 7 minutes. The membranes were blocked with $5 \%$ skim milk in TBS and $0.1 \%$ Tween-20 
(TBST) for 2 hours followed by incubation with the scFvs 4-2 and 22-1 (1 $\mu \mathrm{g} / \mathrm{mL}$ concentration) diluted in TBST for 1 hour. After three washes with TBST, the membranes were incubated with HRP-conjugated rabbit anti-strep tag II antibody (diluted $3 / 10,000$ in 5\% Milk/TBS) (GenScript) at room temperature for 1 hour, followed by washing with TBS-T buffer $3 \times$ for 10 minutes each. The protein was detected and visualized with the ECL detection reagent as described above.

Immunoprecipitation (IP) assay. EBOVHEK293, SUDVHEK293, RESVHEK293, BDBVHEK293, MARVHEK293, LLOVHEK293, and HEK293 cell lysates (containing $10 \mathrm{mg}$ protein) were obtained by lysis with NP40 buffer, protease inhibitor cocktail, and PMSF, and incubated with $25 \mu \mathrm{g}$ of Strep tag-fusion proteins (scFvs 4-2 and 22-1) and left rotating overnight at $4^{\circ} \mathrm{C}$ overnight. Following overnight rotation, then $40 \mu \mathrm{L}$ of Strep 2 Mag beads (IBA GmbH, Germany) were added to the cell lysates and rotated at $4^{\circ} \mathrm{C}$ for 1 hour. The samples were then centrifuged at 3,000 rpm for 1 minute at $4^{\circ} \mathrm{C}$, and the supernatant was discarded. Samples were then washed four times in $1 \mathrm{~mL}$ of Buffer $\mathrm{W}$, centrifuging at $3,000 \mathrm{rpm}$ at $4^{\circ} \mathrm{C}$ for 1 minute and discarding the supernatant each time. Fifty microliters of Laemmli sample buffer was added to the samples, heated to $85^{\circ} \mathrm{C}$ for 2 minutes, centrifuged at 13,000 rpm for 10 minutes, and resolved by SDS-PAGE and Immunoblot as described above. The membranes were blocked with $5 \%$ nonfat dry milk. Membranes (immunoprecipitated complexes) were probed with the mouse (anti-Zaire Ebola antibody 6D8, antiSudan Ebola virus antibody, and anti-His antibody) and rabbit (anti-Reston GP polyclonal antibody, anti-Bundibugyo GP polyclonal antibody, anti-MARV GP polyclonal antibody, and anti-Tai Forest virus GP lgG) as primary antibodies at a dilution of 1:5,000 and the HRP-conjugated donkey anti-mouse and goat anti-rabbit as secondary antibodies at a dilution of 1:3,000 diluted in TBST was added for a 1-hour incubation.

Immunofluorscence assay (IFA). The EBOV, SUDV, BDBV, RESTV, and TAFV viruses were inoculated into 293T cells seeded in eight-well chamber slides (BD Biosciences, San Jose, CA). Forty-eight hours post inoculation infected cells were fixed with $4 \%$ paraformaldehyde solution for 10 minutes at room temperature. After fixation, slides were washed three times with PBS, three minutes per wash, and blocked with PBS-0.1\% BSA. Then cells were washed and incubated for one hour at room temperature with a 1:100 dilution of the ZEBOVGP scFvs 4-2 and 22-1 (500 $\mu \mathrm{g} / \mathrm{mL})$. After incubation, cells were washed three times with PBS, three minutes per wash. Cells were incubated at room temperature with a 1:200 dilution of goat anti-Mouse IgG secondary Antibody, Alexa Fluor 488 (ThermoFisher, Waltham, MA) diluted in blocking buffer (PBS with $0.1 \%$ of BSA) for 1 hour at room temperature. Cells were washed three times as described above, air-dried, and covered with DABCO polyvinvl alcohol mounting medium (Sigma-Aldrich), followed by fluorescent microscope examination.

\section{RESULTS}

Anti-ZEBOVGP scFv antibodies generated from cellfree ribosomal display. To determine whether ribosomal display is suitable for generation of antibodies against filoviruses, we vaccinated mice with VLPs consisting of EBOV VP40 and GP. The use of VLPs as a protective vaccine is wellestablished, as we and others have shown. ${ }^{24,29}$
For ribosome-displayed scFv antibody libraries, the immunoglobulin $\mathrm{VH}$ and $\mathrm{VL}$ regions joined to a 20 amino acid flexible linker $\left(\left[\mathrm{G}_{4} \mathrm{~S}\right]_{4}\right)$ were constructed using RNA isolated from the spleen of mouse \#1 (Figures 1 and 2 and Supplemental Table 1). Complementary DNA was synthesized using a single consensus primer MVKR and then pooled and directly used as a template for PCR amplification of the $\mathrm{VH}$ and $\mathrm{VL}$ chain gene fragments. The gene fragments from mouse were pooled in one reaction tube together with primers that contain overlapping linker sequences which allow the $\mathrm{VH}$ and $\mathrm{VL}$ gene segments to be assembled by overlap extension to form strep tag-conjugated scFvs (Figure 2). The amplified PCR product was of the expected size of about $900 \mathrm{bp}$. The final DNA template encoding the library flanked by a T7 site was used in an in vitro ribosome display with a single selection step with recombinant EBOV GP as outlined in Figure 1.

After selection, the mRNA was recovered as DNA from the bound ternary complexes by in situ RT-PCR (Figure 2B and C). The PCR product was then cloned into PGEM-T vector. DNA from 10 randomly chosen clones from the libraries was sequenced and were aligned using Clustal Omega. Their amino acid sequences were deduced and three complementary determining regions (CDRs) and four framework (FW) regions were identified in each of the $\mathrm{VH}$ and $\mathrm{VL}$ chain fragments, which are linked by a 20 amino acid $\left(\mathrm{G}_{4} \mathrm{~S}\right)_{4}$ linker. Following alignment with each other, significant diversity in the $\mathrm{VH}$ and VL chain was observed especially in the CDRs. Variability was also noted in the framework regions (FRs). No two clones had identical VH or VL fragments. The aligned amino acid sequences of 10 clones from library are shown in Supplemental Figure 2. The FRs and CDRs were determined by the IMGT information system (http://imgt.cines.fr/) (IMGT $\left.{ }^{\circledR} / \mathrm{N}-\mathrm{Quest}\right) .{ }^{30}$ The length of CDRs was variable. The length of CDR1 VH, with an average length of eight amino acid residues, CDR2VH with an average length of eight residues, CDR3 $\mathrm{VH}$ ranged from 10 to 18 amino acid residues, with an average length of 13 residues, CDR1 VL with an average length of nine residues, while three amino acid residues were found in CDR2VL and nine amino acid residues were found in CDR3 VL.

After expression of scFv clones, samples were harvested, lysed, purified, and fractions were analyzed by SDS-PAGE (to confirm the integrity and purity) followed by Western blot. The purified protein gave a prominent band of about $33 \mathrm{kDa}$ (Figure 3A).

Specificity, cross-reactivity, and affinity of GP-specific scFvs. The scFvs were screened in ELISA binding assays for their specificity and cross-reactivity with recombinant GPs of the other known filoviruses in the genus Ebolavirus (SUDV, TAFV, BDBV, and RESTV) and MARV. Several different profiles for the cross-reactivities of these antibodies were found (Supplemental Table 2 and Figure $3 \mathrm{~B}$ and C). Two scFvs (EBOVGP4-2 and EBOVGP22-1) reacted strongly with all tested GPs of Ebolavirus and Marburg species. Six scFvs (EBOVGP3-2, EBOVGP13-1, EBOVGP16-1, EBOVGP18-1, EBOVGP35-2, and EBOVGP35-1) bound weakly to GPs of some viruses. In addition to EBOV and EBOVGP scFv3-2 reacted only to TAFV and MARV, and EBOVGP scFv27-5 did not react with any GPs. Importantly, these different reactivity profiles enabled us to distinguish the known Ebolavirus species by using scFvs (4-2 and 22-1), as shown in Figure 3B and $\mathrm{C}$ and Supplemental Table 2. Representative scFvs for each obtained cross-reactivity profile showing the highest OD 


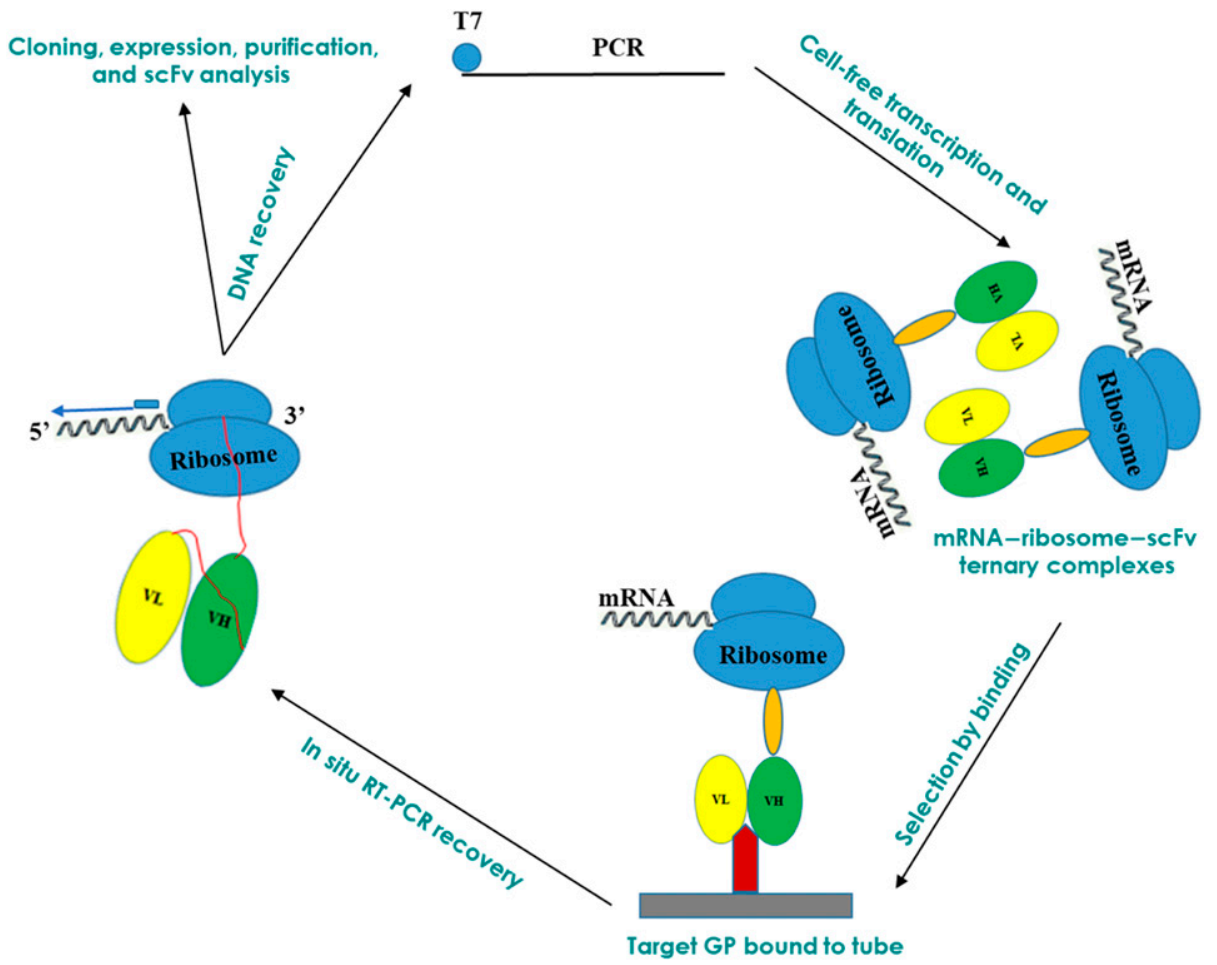

FIGURE 1. Graphical overview: scFVs were generated using cell-free ribosomal display from mice immunized with filovirus viruslike particles. scFvs $=$ single-chain Fvs. This figure appears in color at www.ajtmh.org.

values were selected for dose-response relationship studies based on the overlap of common motifs (data not shown).

Dose-response curves were determined for scFvs EBOVGP4-2 and EBOVGP22-1 (Figure 4A and B),
(Supplemental Tables 3-6, highlighted rows). Both scFvs bound tightly to all six GPs, with $\mathrm{EC}_{50}$ s ranging from 4 to $12 \mu \mathrm{g} /$ $\mathrm{mL}$. EBOVGP scFv4-2 and EBOVGP scFv22-1 showed the strongest binding to all six ebolavirus species, with $\mathrm{EC}_{50} \mathrm{~S}$

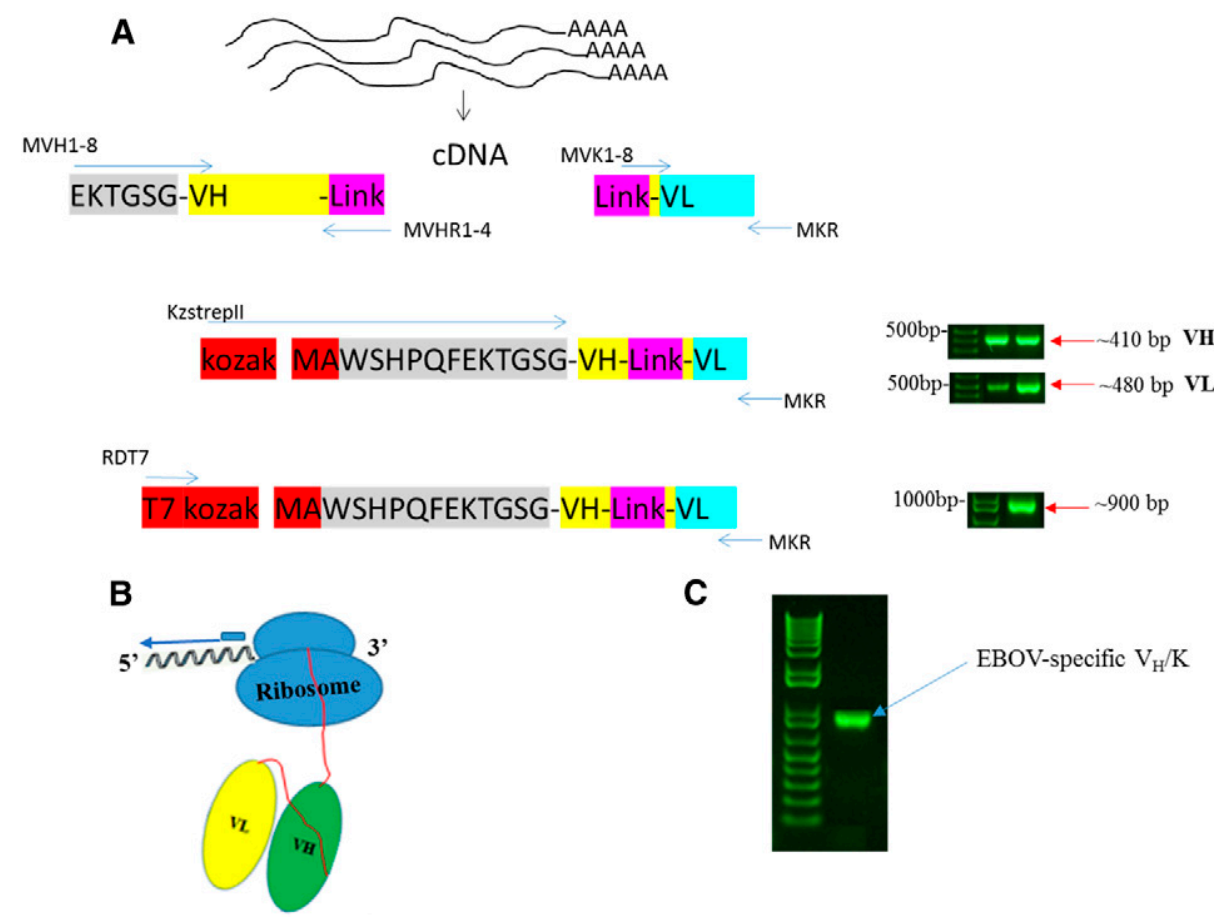

FIGURE 2. (A) Schematic representation of making an scFv library from mouse spleen. (B) Schematic of the stalled antibody-ribosome-mRNA complex and position of primers used for RT-PCR recovery in the 1st cycle of ribosome display. T7 is the $5^{\prime}$ primer and MKR is the $3^{\prime}$ primer. (C) Analysis of RT-PCR recovery of $\mathrm{V}_{\mathrm{H}} / \mathrm{K}$ cDNA in the 1 st cycle. $\mathrm{V}_{\mathrm{H}} / \mathrm{K}$ complexes were bound to EBOV GP coated to the PCR tube through 1 st cycle of selection and recovery. cDNA = complementary DNA; Ebola virus = EBOV; GP = glycoprotein; RT-PCR = reverse transcription PCR. This figure appears in color at www.ajtmh.org. 
A

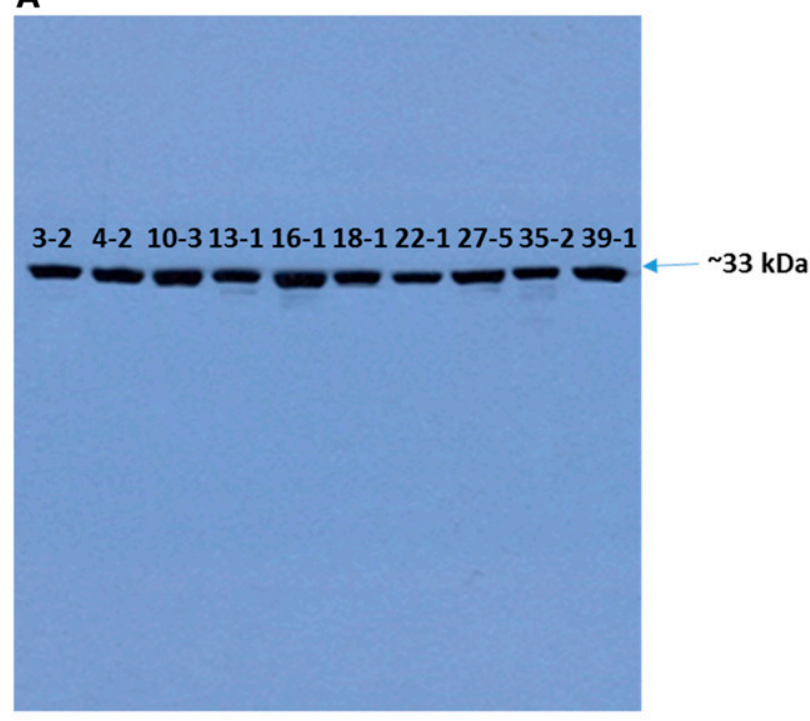

B

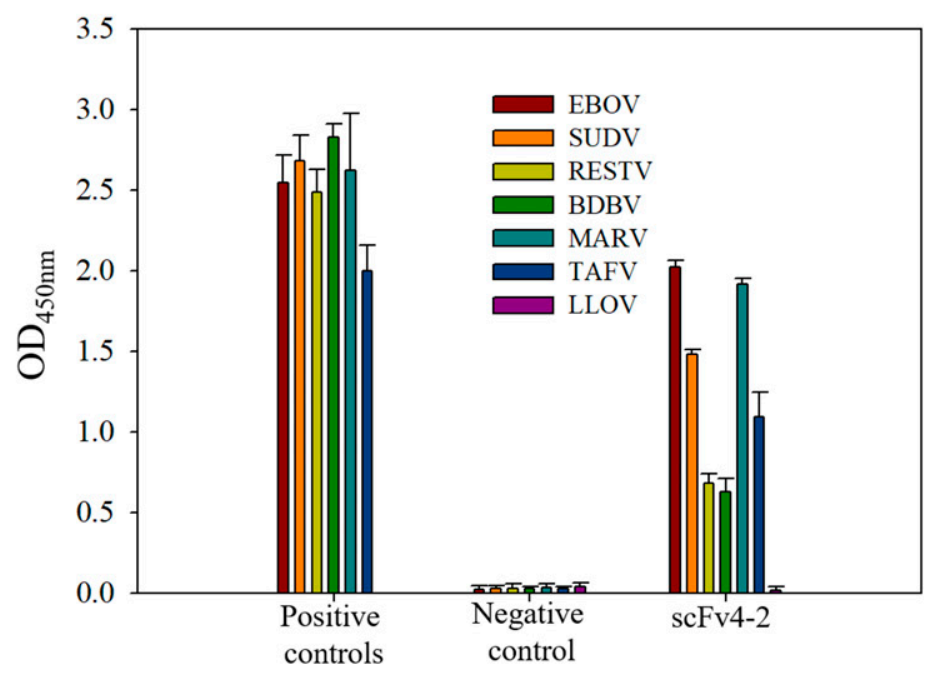

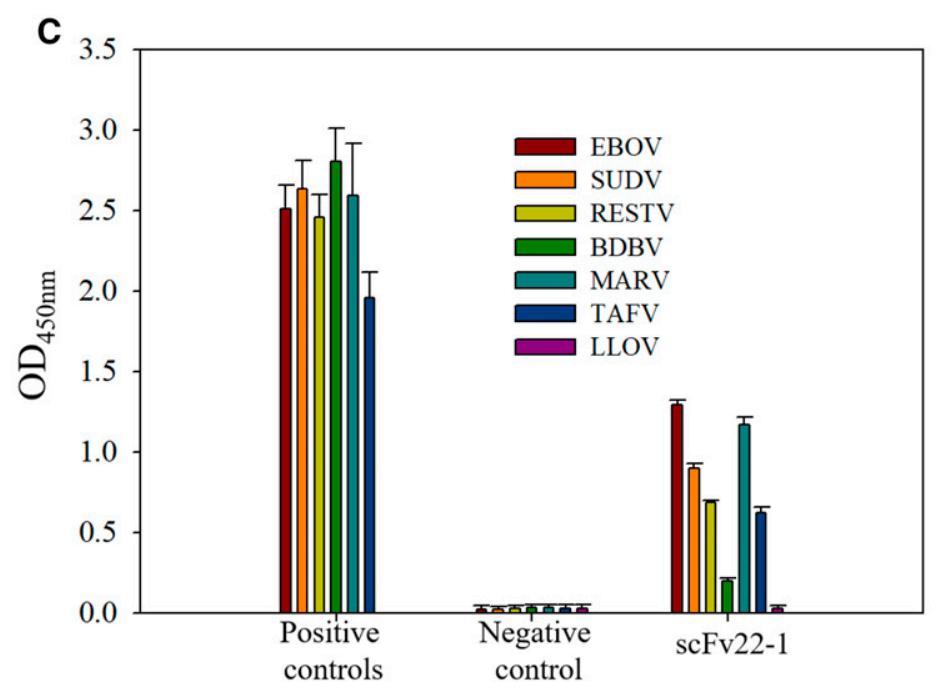

FIGURE 3. (A) Western blot of purified scFvs (3-2, 4-2, 10-3, 13-1, 16-1, 18-1 22-1, 27-5, 35-2, and 39-1). ELISA results for purified novel antiEBOV scFv4-2 (B) and scFv22-1 (C) (generated by ribosome display), commercial monoclonal (mouse anti-EBOV antibody 6D8 and mouse antiSUDV ebola virus antibody), and polyclonal (rabbit anti-RESTV GP, rabbit anti-BDBV GP, rabbit anti-MARV GP, and rabbit anti-TAFV GP) antibodies as positive controls, and an scFv negative control (mouse anti-PilB scFv21 antibody). BDBV = Bundibugyo virus; EBOV = Ebola virus; GP = glycoprotein; MARV = Marburg virus; SUDV = Sudan virus; TAFV = Tai Forest virus. This figure appears in color at www.ajtmh.org.

below 9 and $12 \mu \mathrm{g} / \mathrm{mL}$, respectively. The EBOVGP-scFv 4-2 demonstrated almost 2-fold enhanced binding compared to EBOVGP-scFv 22-1. Binding constant (KD) determinations were estimated by fitting a dose-response curve, and the KD estimates for the scFvs against all six GPs are shown in Supplemental Table 7. The highest affinity scFv was the anti-EBOVGP scFv4-2, which was about 2-fold greater toward all six GPs than the antiEBOVGP scFv22-1.

To assess the ability of the anti-EBOVGP scFvs to recognize the six filovirus GPs in a denatured format, Western blotting was performed on purified GPs. Anti-EBOVGP scFvs 4-2 and 22-1 binding to ebolavirus GPs was completely lost on denaturation,

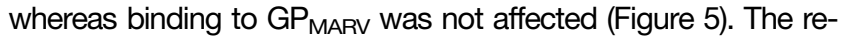
activities of these GP-specific scFvs were further tested by IP analysis using lysates of filovirus GP expressed in 293T cells. The results demonstrated that the anti-EBOVGP scFvs were able to immunoprecipitate filovirus GPs, confirming the ELISA data (Figure 6).
An IFA was performed to confirm the recognition of scFvs with the proteins of $\mathrm{GP}_{\mathrm{EBOV}}, \mathrm{GP}_{\text {SUDV }}$, and $\mathrm{GP}_{\mathrm{MARV}}$ in its natural membrane-associated trimeric structure with 293T cells transfected with the $\mathrm{GP}_{\mathrm{EBOV}^{-}}, \mathrm{GP}_{\mathrm{SUDV}^{-}}$, and $\mathrm{GP}_{\mathrm{MARV}^{-}}$ expressing plasmids (Figure 7). The results demonstrated that these two scFvs (4-2 and 22-1) effectively recognized and stained the $\mathrm{GP}_{\text {EBOV }}, \mathrm{GP}_{\text {SUDV }}$, and $\mathrm{GP}_{\mathrm{MARV}}$ expressed in the 293T cells. The staining ability of scFv4-2 was superior to scFv22-1, and these are comparable to the results of positive controls in the IFA.

\section{DISCUSSION}

The latest Ebola virus disease outbreak in West Africa has highlighted the urgent need for rapid and effective diagnostics against filoviruses. In this study, we have generated a panel of mouse scFvs using cell-free ribosome display with a range of 


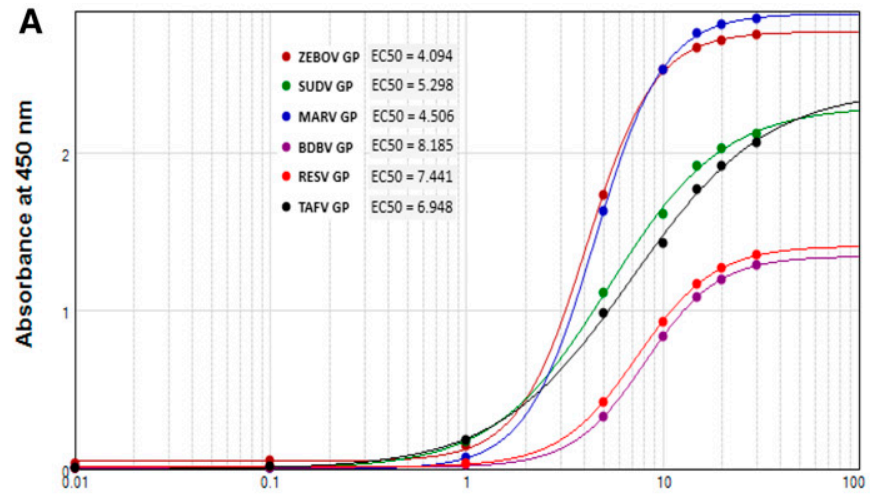

$\operatorname{scFv4-2}(\mu \mathrm{g} / \mathrm{ml})$

\section{C}

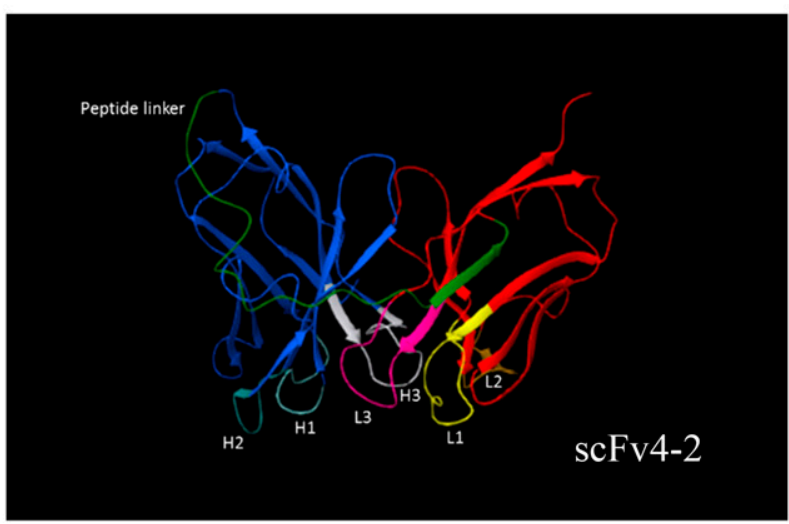

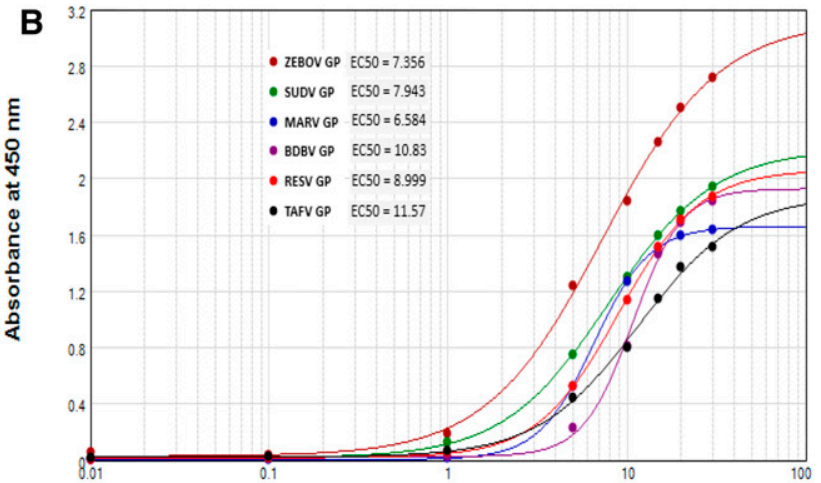

$\mathrm{scFv22-1}(\mu \mathrm{g} / \mathrm{ml})$

D

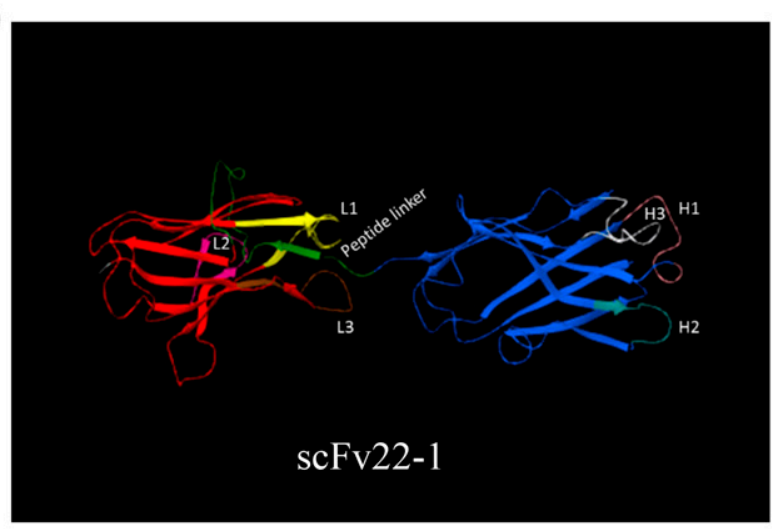

FIGURE 4. (A and B) Reactivity of mouse ZEBOV GP scFvs (4-2 and 22-1) to filovirus glycoproteins and dose response binding of the indicated antibodies to GPs of EBOV, SUDV, MARV, BDBV, RESTV, and TAFV. Values are optical density at $450 \mathrm{~nm}\left(\mathrm{OD}_{450}\right)$ values from three ELISA experiments performed over the indicated range of antibody concentrations. The EC50s (in micrograms per milliliter) for binding of each antibody to the respective antigen are shown in each panel. Predicted ribbon representation ( $\mathbf{C}$ and $\mathbf{D}$ ) of anti-ZEBOV scFv antibody molecules (4-2 and 22-1) in $3 \mathrm{D}$ mode. $\mathrm{BDBV}=\mathrm{Bundibugyo}$ virus; $\mathrm{EBOV}=$ Ebola virus; $\mathrm{GP}=$ glycoprotein; $\mathrm{MARV}=$ Marburg virus; $\mathrm{SUDV}=\mathrm{Sudan}$ virus; $\mathrm{TAFV}=\mathrm{Tai}$ Forest virus; scFvs = single-chain Fvs. This figure appears in color at www.ajtmh.org.

cross-reactivity to five species of ebolavirus and to marburgvirus. Two of these scFvs can cross-react to all six known pathogenic filoviruses, representing pan-filovirus binding. This is the first report of the use of cell-free ribosome display to generate broadly reactive scFvs against filoviruses.
Of the 10 anti-EBOV GP scFvs tested by ELISA, there were two that were highly cross-reactive to all ebola- and marburgvirus GPs (Supplemental Table 2 and Figure 3B and C), but not LLOV GP, suggesting that they are indeed antigen specific. Holtsberg et al. ${ }^{31}$ demonstrated that a single MAb (m21D10) reactive to three GP (EBOV, SUDV, and MARV)
A

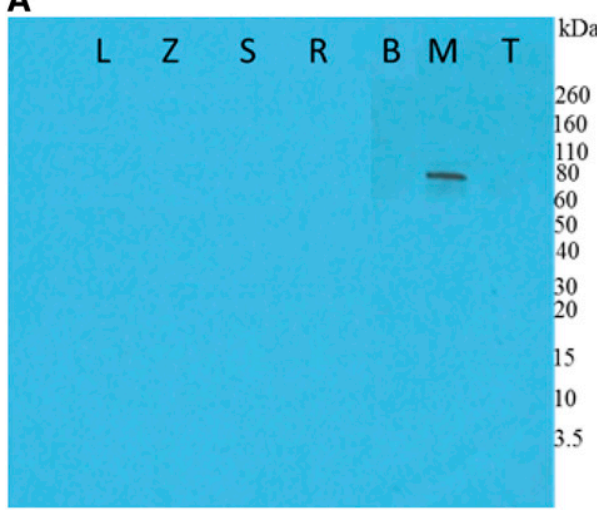

B

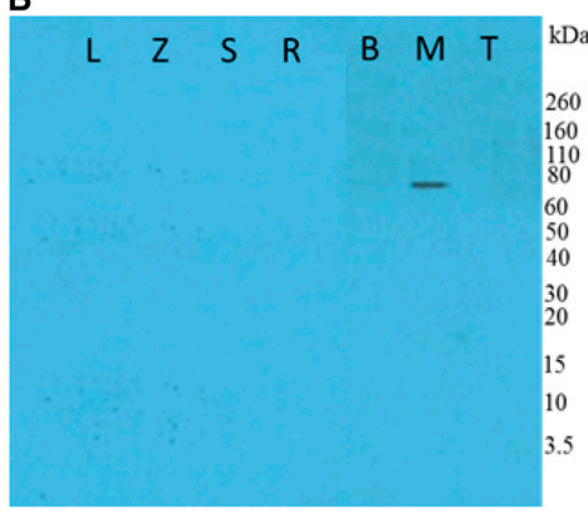

FIGURE 5. Denaturation eliminates binding of scFV4-2 and scFv 22-1 for the ebolavirus GPs. The purified proteins of LLOV, (L) EBOV (Z), SUDV (S), RESTV (R), BDBV (B), MARV (M), and TAFV (T) GPs without TM were probed with soluble scFvs 4-2 and 22-1. BDBV = Bundibugyo virus; EBOV = Ebola virus; GP = glycoprotein; MARV = Marburg virus; SUDV = Sudan virus; TAFV = Tai Forest virus; scFvs = single-chain Fvs. This figure appears in color at www.ajtmh.org. 
A

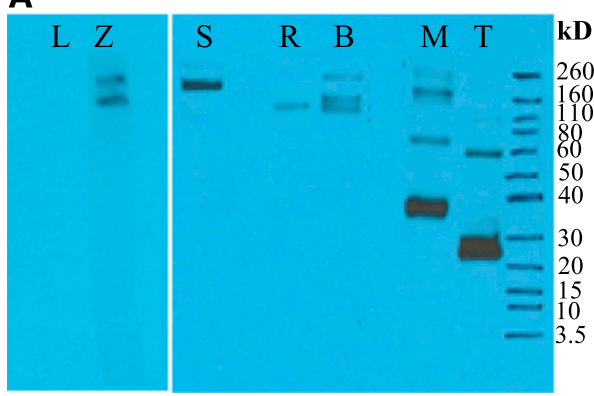

B

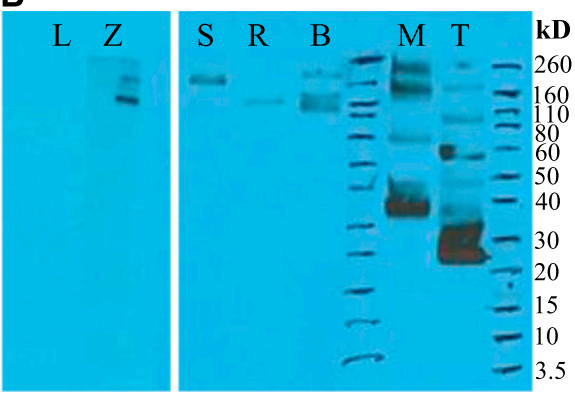

FigurE 6. Immunoprecipitation of filovirus GPs by scFvs 4-2 and 22-1. The lysates of LLOV (L)-, EBOV (Z)-, SUDV (S)-, RESTV (R)-, BDBV (B)-, MARV (M)-, and TAFV (T)-infected 293T cells were immunoprecipitated with soluble scFvs 4-2 and 22-1 followed by SDS-PAGE analysis and Western blot. $\mathrm{BDBV}=$ Bundibugyo virus; $\mathrm{EBOV}=$ Ebola virus; $\mathrm{GP}=$ glycoprotein; $\mathrm{MARV}=$ Marburg virus; $\mathrm{SUDV}=\mathrm{Sudan}$ virus; $\mathrm{TAFV}=\mathrm{Tai}$ Forest virus; scFvs = single-chain Fvs. This figure appears in color at www.ajtmh.org.

molecules and six clones reactive to both EBOV and SUDV but nonreactive to MARV were identified. Keck et al. ${ }^{32}$ identified a set of broadly reactive pan-ebolavirus MAbs with strong binding to EBOV, SUDV, BDBV, and RESTV GP; two MAbs also exhibited weak binding to MARV GP. Other studies have also found broadly cross-reactive ebolavirus GP antibody (PMID: 28525756 and 28525755). Our binding data showed that the cross-reactive scFvs, 4-2 and 22-1, exhibited moderate to higher binding with submicromolar $\mathrm{EC}_{50}$ s to five ebolavirus GP and a marburgvirus GP (Figure 4A and B). Our results are comparable to the results from the dose-dependent binding of mAb m21D10 to EBOV, SUDV, and MARV GPs ${ }^{31}$ and FVM04, exhibited balanced binding at subnanomolar concentrations to four ebolavirus species tested, shows weak binding to MARV. ${ }^{32}$ scFv4-2 antibody showed an approximate 2-fold high affinity to all GPs in comparison to the scFv22-1 antibody (Supplemental Table 7 and Figure 7). Rodríguez-Martínez et al. ${ }^{33}$ reported that the scFv$13 \mathrm{C} 6 \mathrm{mAb}$ exhibited the highest EBOV GP-binding activity, followed by scFv-13F6 and Fab-KZ52 in ELISA experiments.

Western blot suggests that the conformational epitopes recognized by these scFvs are completely destroyed when GPs are denaturated (Figure 5). Interestingly, these scFvs recognized MARV GP, suggesting that these scFvs bind to different epitopes (data not shown). These scFvs bound to all six native GPs in an ELISA, but not a Western blot (except for MARV GP), suggesting these scFvs can recognize GPs in its natural conformation and binds to conformational epitopes.

On IP analysis using lysates of filovirus GPs produced in 293T cells (Figure 6), the cross-reactivity profiles and virus specificities were similar to those obtained by ELISA. These scFvs, cross-reactive to all known ebolaviruses, may be very important for the development of Ebola and MARV antigen detection assays.

Although a previous study demonstrated in vivo efficacy of anti-marburgvirus GP scFvs, ${ }^{34}$ this is the first report of an scFv antibody (4-2 or 22-1) that exhibits broadly cross-reactive activity against filovirus GP. These scFvs also have the potential for the identification of as yet unknown Ebolavirus species. During the first reported outbreak of BDBV, initial diagnosis was made using an antigen capture ELISA using a combination of anti-ZEBOV, SUDV, and RESTV mAbs as well as hyperimmune polyclonal anti-EBOV rabbit serum, whereas the specimens were initially negative when tested by RT-PCR. ${ }^{35}$ This highlights the value of scFvs to potentially identify new Ebola and MARV species. The scFvs generated in

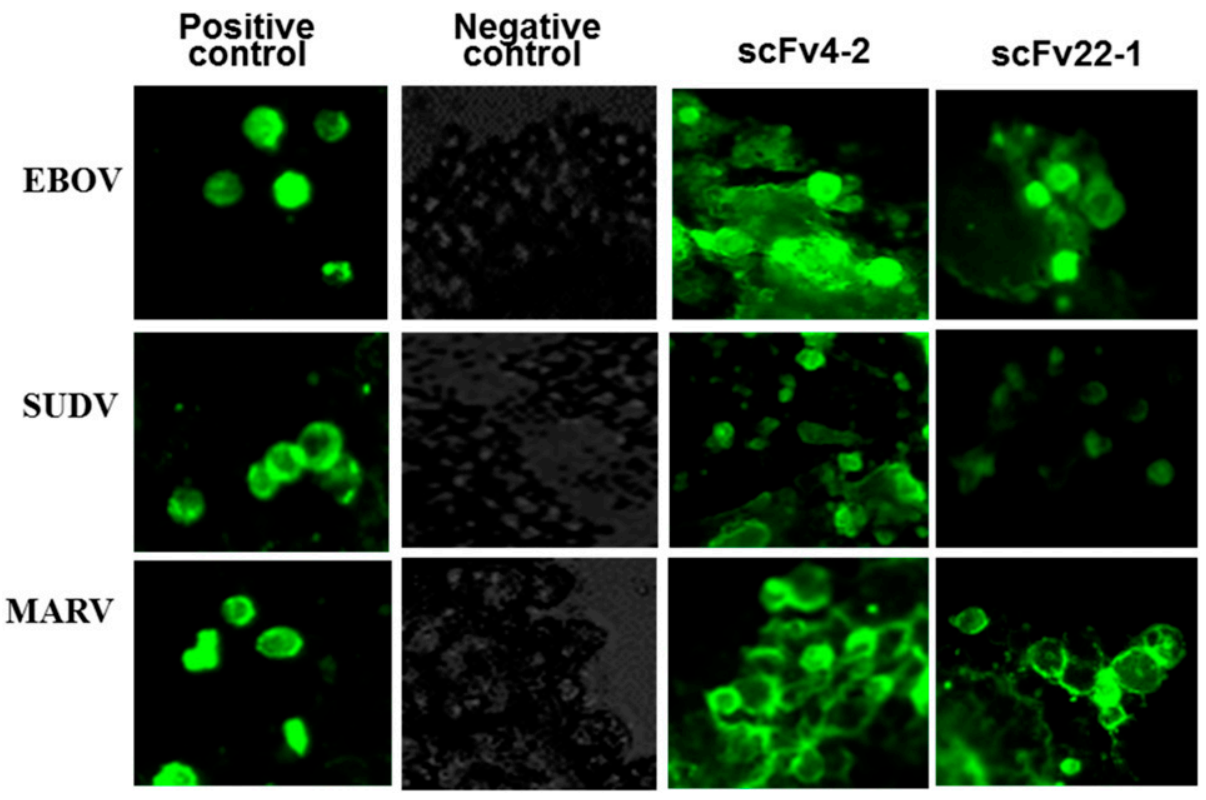

FIGURE 7. The scFvs $4-2$ and $22-1$, and positive controls recognize the natural forms of GP1,2ZEBOV, GP1,2sUDV, and GP1,2 glycoprotein; scFvs = single-chain Fvs. This figure appears in color at www.ajtmh.org. 
this study may therefore offer some advantage in generating a highly sensitive assay for diagnostic use.

In summary, a panel of EBOVGP-specific scFvs from ribosome display and their cross-reactivity profiles to all known human pathogenic filoviruses were established. This is the first report on ribosome display scFvs that can detect all known ebolaviruses. This method is inexpensive, rapid, and can be used to quickly develop repertoires of high-affinity antibodies. These broadly cross-reactive scFvs produced in this study have great diagnostic potential. Our next steps will be testing these scFv antibodies with live viruses.

Received August 10, 2018. Accepted for publication March 16, 2019. Published online May 6, 2019.

Note: Supplemental tables and figures appear at www.ajtmh.org.

Acknowledgments: This project is supported in part by HDTRA1-151-0061 (S. B. B.). The project or effort depicted was or is sponsored by the Department of the Defense, Defense Threat Reduction Agency. The content of the information does not necessarily reflect the position or the policy of the federal government and no official endorsement should be inferred.

Authors' addresses: Adinarayana Kunamneni and Ravi Durvasula, Department of Medicine, Loyola University Medical Center, Chicago, IL, E-mails: akunamneni@luc.edu and ravi.durvasula@lumc.edu. Elizabeth C. Clarke, Chunyan Ye, Steven B. Bradfute, Department of Internal Medicine, Center for Global Health, University of New Mexico, Albuquerque, NM, E-mails: eclarke@salud.unm.edu, cye@salud. unm.edu, and sbradfute@salud.unm.edu.

This is an open-access article distributed under the terms of the Creative Commons Attribution License, which permits unrestricted use, distribution, and reproduction in any medium, provided the original author and source are credited.

\section{REFERENCES}

1. Whitehouse CA, Bavari S, Perkins MD, 2015. United States FDA's emergency use authorization of Ebola virus diagnostics: current impact and lessons for the future. Expert Rev Mol Diagn 15: 1231-1235.

2. Anthony SM, Bradfute SB, 2015. Filoviruses: one of these things is (not) like the other. Viruses 7: 5172-5190.

3. WHO, 2015. Urgently Needed: Rapid, Sensitive, Safe and Simple Ebola Diagnostic Tests. Available at: www.who.int/ mediacentre/news/ebola/18-november-2014-diagnostics/en.

4. Kugelman JR et al., 2015 Evaluation of the potential impact of Ebola virus genomic drift on the efficacy of sequence-based candidate therapeutics. mBio 6: e02227-14.

5. Marzi A et al., 2018 Recently identified mutations in the ebola virus-makona genome do not alter pathogenicity in animal models. Cell Rep 23: 1806-1816.

6. Connolly BM, Steele KE, Davis KJ, Geisbert TW, Kell WM, Jaax NK, Jahrling PB, 1999. Pathogenesis of experimental Ebola virus infection in guinea pigs. J Infect Dis 179 (Suppl 1): S203-S217.

7. Hevey M, Negley D, Geisbert J, Jahrling P, Schmaljohn A, 1997 Antigenicity and vaccine potential of Marburg virus glycoprotein expressed by baculovirus recombinants. Virology 239: 206-216.

8. Bray M, Davis K, Geisbert T, Schmaljohn C, Huggins J, 1998. A mouse model for evaluation of prophylaxis and therapy of Ebola hemorrhagic fever. J Infect Dis 178: 651-661.

9. Takada A, Feldmann H, Stroeher U, Bray M, Watanabe S, Ito $H$, McGregor M, Kawaoka Y, 2003. Identification of protective epitopes on ebola virus glycoprotein at the single amino acid level by using recombinant vesicular stomatitis viruses. J Virol 77: 1069-1074.

10. Kajihara $M$, Nakayama $E$, Marzi A, Igarashi $M$, Feldmann $H$, Takada A, 2013. Novel mutations in Marburg virus glycoprotein associated with viral evasion from antibody mediated immune pressure. J Gen Virol 94: 876-883.

11. He M, Taussig MJ, 2002. Ribosome display: cell-free protein display technology. Brief Funct Genomic Proteomic 1: 204-212.
12. Schaffitzel C, Hanes J, Jermutus L, Plückthun A, 1999. Ribosome display: an in vitro method for selection and evolution of antibodies from libraries. $J$ Immunol Methods 231: 119-135.

13. Kunamneni A, Ye C, Bradfute SB, Durvasula R, 2018. Ribosome display for the rapid generation of high-affinity Zika-neutralizing single-chain antibodies. PLoS One 13: e0205743.

14. Luginbuhl B, Kanyo Z, Jones RM, Fletterick RJ, Prusiner SB, Cohen FE, Williamson RA, Burton DR, Plückthun A, 2006. Directed evolution of an anti-prion protein ScFv fragment to an affinity of 1 pM and its structural interpretation. J Mol Biol 363: 75-97.

15. He M, Khan F, 2005. Ribosome display: next-generation display technologies for production of antibodies in vitro. Expert Rev Proteomics 2: 421-430.

16. Gu L, Li C, Aach J, Hill DE, Vidal M, Church GM, 2014. Multiplex single-molecule interaction profiling of DNA-barcoded proteins. Nature 515: 554-557.

17. Cong C, Yu X, He Y, Dai Y, Zhang Y, Wang M, He M, 2016. Cellfree ribosome display and selection of antibodies on arrayed antigens. Proteomics 16: 1291-1296.

18. Bird RE, Hardman KD, Jacobson JW, Johnson S, Kaufman BM, Lee SM, Lee T, Pope SH, Riordan GS, Whitlow M, 1988. Singlechain antigen-binding proteins. Science 242: 423-426.

19. Huston JS et al., 1988. Protein engineering of antibody binding sites: recovery of specific activity in an anti-digoxin single-chain Fv analogue produced in Escherichia coli. Proc Natl Acad Sci U S A 85: 5879-5883.

20. Shen Z, Stryker GA, Mernaugh RL, Yu L, Yan H, Zeng X, 2005. Single-chain fragment variable antibody piezoimmunosensors. Anal Chem 77: 797-805.

21. Padlan EA, 1994. Anatomy of the antibody molecule. Mol Immunol 31: 169-217.

22. National Research Council, 1996. National Science Education Standards. Washington, DC: The National Academies Press, 272.

23. Markiv A, Anani B, Durvasula RV, Kang AS, 2011. Module based antibody engineering: a novel synthetic REDantibody. J Immunol Methods 364: 40-49.

24. Ayithan N, Bradfute SB, Anthony SM, Stuthman KS, Dye JM, Bavari S, Bray M, Ozato K, 2014. Ebola virus-like particles stimulate type I interferons and proinflammatory cytokine expression through the toll-like receptor and interferon signaling pathways. J Interferon Cytokine Res 34: 79-89.

25. Ayithan N, Bradfute SB, Anthony SM, Stuthman KS, Bavari S, Bray M, Ozato K, 2015. Virus-like particles activate type I interferon pathways to facilitate post-exposure protection against ebola virus infection. PloS One. 2015;10:e0118345.

26. Azizi A, Arora A, Markiv A, Lampe DJ, Miller TA, Kang AS, 2012. Ribosome display of combinatorial antibody libraries derived from mice immunized with heat-killed Xylella fastidiosa and the selection of MopB-specific single-chain antibodies. Appl Environ Microbiol 78: 2638-2647.

27. He M, Taussig MJ, 2007. Eukaryotic ribosome display with in situ DNA recovery. Nat Methods 4: 281-288.

28. Gasteiger E, Gattiker A, Hoogland C, Ivanyi I, Appel RD, Bairoch A, 2003. ExPASy: the proteomics server for in-depth protein knowledge and analysis. Nucleic Acids Res 31: 3784-3788.

29. Bradfute SB, Anthony SM, Stuthman KS, Ayithan N, Tailor P, Shaia Cl, Bray M, Ozato K, Bavari S, 2015. Mechanisms of immunity in post-exposure vaccination against ebola virus infection. PloS One 10: e0118434.

30. Giudicelli V, Chaume D, Lefranc MP, 2004. IMGT/N-QUEST, an integrated software program for immunoglobulin and $T$ cell receptor V-J and V-D-J rearrangement analysis. Nucleic Acids Res 32 (Web Server issue): W435-W440.

31. Holtsberg FW et al., 2015. Pan-ebolavirus and pan-filovirus mouse monoclonal antibodies: protection against ebola and Sudan viruses. J Virol 90: 266-278.

32. Keck ZY et al., 2015. Macaque monoclonal antibodies targeting novel conserved epitopes within filovirus glycoprotein. $J$ Virol 90: 279-291.

33. Rodríguez-Martínez LM et al., 2015. Antibody derived peptides for detection of ebola virus glycoprotein. PLoS One 10: e0135859.

34. Froude JW et al., 2017. Generation and characterization of protective antibodies to Marburg virus. MAbs 9: 696-703.

35. Towner JS et al., 2008. Newly discovered ebola virus associated with hemorrhagic fever outbreak in Uganda. PLoS Pathog 4: e1000212. 